\title{
Decreased aortic growth and middle aortic syndrome in patients with neuroblastoma after radiation therapy
}

\author{
Elizabeth J. Sutton • Ricky T. Tong • Amy M. Gillis • Tobias D. Henning • \\ Vivian A. Weinberg • Sophie Boddington • Daphne A. Haas-Kogan • \\ Katherine Matthay $\cdot$ Vinil Sha $\cdot$ Charles Gooding $\cdot$ Fergus V. Coakley • \\ Heike Daldrup-Link
}

Received: 25 February 2009 /Revised: 26 May 2009 / Accepted: 22 June 2009/Published online: 18 September 2009

(C) The Author(s) 2009. This article is published with open access at Springerlink.com

\begin{abstract}
Background Long-term CT follow-up studies are required in pediatric patients who have received intraoperative radiation therapy (IORT) and external beam radiation therapy (EBRT) to assess vascular toxicities and to determine the exact complication rate.

Objective To analyze with CT the effects of radiation therapy (RT) on the growth of the aorta in neuroblastoma patients. Materials and methods Abdominal CT scans of 31 patients with intraabdominal neuroblastoma (stage II-IV), treated with
\end{abstract}

\section{E. J. Sutton}

Department of Radiology, Mount Auburn Hospital,

Harvard University,

Cambridge, MA, USA

R. T. Tong

Department of Medicine, Stanford University,

Palo Alto, CA, USA

\section{A. M. Gillis · D. A. Haas-Kogan}

Department of Radiation Oncology, University of California,

San Francisco,

San Francisco, CA, USA

E. J. Sutton · T. D. Henning $\cdot$ S. Boddington $\cdot$ V. Sha $\cdot$

C. Gooding $\cdot$ F. V. Coakley $\cdot$ H. Daldrup-Link $(\triangle)$

Department of Radiology, University of California, San Francisco,

505 Parnassus Ave.,

San Francisco, CA 94131, USA

e-mail: daldrup@radiology.ucsf.edu

V. A. Weinberg

Comprehensive Cancer Center, Biostatistics Core,

University of California, San Francisco,

San Francisco, CA, USA

K. Matthay

Department of Pediatrics, University of California, San Francisco,

San Francisco, CA, USA
RT (20 IORT \pm EBRT, 11 EBRT alone), were analyzed retrospectively. The diameter of the abdominal aorta was measured before and after RT. These data were compared to normal and predicted normal aortic diameters of children, according to the model of Fitzgerald, Donaldson and Poznanski (aortic diameter in centimeters $=0.844+0.0599 \times$ age in years), and to the diameters of a control group of children who had not undergone RT. Statistical analyses for the primary aims were performed using the chi-squared test, $t$-test, Mann-Whitney test, nonparametric Wilcoxon matched-pairs test and analysis of variance for repeated measures. Clinical files and imaging studies were evaluated for signs of late vascular complications of neuroblastoma patients who had received RT.

Results The mean diameter before and after RT and the growth of the aorta were significantly lower than expected in patients with neuroblastoma $(P<0.05$ for each) and when compared to the growth in a control group with normal and nonirradiated aortas. Among the patients who had received $\mathrm{RT}$, there was no difference due to the type of RT. Seven patients from the IORT \pm EBRT group developed vascular complications, which included hypertension (five), middle aortic syndrome (two), death due to mesenteric ischemia (one) and critical aortic stenosis, which required aortic bypass surgery (two).

Conclusion Patients with neuroblastoma who had received RT showed impaired growth of the abdominal aorta. Significant long-term vascular complications occurred in seven patients who received IORT \pm EBRT. Thus, CT evaluation of patients with neuroblastoma who receive RT should include not only reports of changes in tumor extension, but also documentation of perfusion, and the size and growth of the aorta and its branches over time.

Keywords Neuroblastoma - Middle aortic syndrome . Therapy complications $\cdot$ Intraoperative irradiation $\cdot$ Children 


\section{Introduction}

Neuroblastoma is the most common extracranial solid tumor in children and often presents as a large abdominal mass that encases major blood vessels [1-3]. Treatment of neuroblastoma depends on clinical and biological risk factors. Multimodality therapy, including surgery, chemotherapy and adjuvant radiotherapy, followed by myeloablative therapy and peripheral blood stem cell transplantation appear most effective in the treatment of high-risk disease $[4,5]$.

Adjuvant radiotherapy has proven effective for local control in the treatment of high-risk neuroblastomas (stage III and IV) [6, 7]. The role of adjuvant radiotherapy continues to evolve as physicians balance treatment benefits against long-term side effects, which are particularly severe in children [6-10]. External beam radiation therapy (EBRT) has sometimes been supplemented or replaced by intraoperative radiation therapy (IORT) in these patients. IORT allows the administration of high-dose radiation to a limited field, thereby maximizing tumoricidal radiation effects and minimizing toxicity to normal tissue [11]. However, since neuroblastomas often encase abdominal vessels, these vessels are also exposed to relatively high radiation doses. The majority of reports on IORT are limited to short- and intermediate-term follow-up studies in adults and canine models [12-16]. However, several studies have demonstrated the efficacy of IORT for achieving local control in children [7, 11, 17-19]. Canine models have demonstrated deleterious effects of IORT on the aorta and branch arteries including marked vascular narrowing in long-term followup studies exceeding 5 years $[13,20]$.

We recently encountered three children with intraabdominal high-risk neuroblastoma at our institution who developed a severe middle aortic syndrome (MAS) several years after successful multimodal treatment of their neuroblastoma (surgery, chemotherapy and IORT). The MAS led to death due to mesenteric ischemia in one patient and required an arterial bypass surgery in the other two patients. MAS is a clinicopathological definition referring to isolated disease of the abdominal aorta comprising significant proximal tubular narrowing with stenosis that results in uncontrollable hypertension and deteriorating renal function [21-23].

To the best of our knowledge, no studies exist that have evaluated the effects of IORT and/or EBRT on the abdominal aorta and tributaries in patients with neuroblastoma. Thus, the purpose of our study was to analyze with CT the effects of multimodality therapy including IORT, with or without EBRT, or EBRT alone on the growth of the aorta in patients with intraabdominal neuroblastoma and to determine if vascular complications occurred in these patients.

\section{Materials and methods}

The Committee of Human Research at our institution approved this retrospective study and did not require patient informed consent. All investigators complied with Health Insurance Portability and Accountability Act regulations.

\section{Patients}

In a retrospective evaluation of our pediatric oncology patient population, we investigated the abdominal aorta of patients with neuroblastoma treated with radiation therapy (RT) on CT scans. The inclusion criteria for this study were patients with neuroblastoma and an intraabdominal primary tumor who were treated with IORT and/or EBRT and who had available CT imaging before RT. A total of 69 neuroblastoma patients treated consecutively between 1993 and 2005 received RT, but 25 had to be excluded due to lack of available imaging studies. A total of 44 patients had at least one CT scan available, including 29 who had IORT with or without EBRT, and 15 who had EBRT without IORT (Table 1), and 31 of these 44 (10 IORT, 10 IORT + EBRT, 11 EBRT) had imaging studies before RT and were thus analyzed; of these, 25 had scans both before and after RT.

\section{IORT and EBRT}

The 20 patients with pre-RT scans receiving IORT were treated between 1993 and 2004 with only two treated prior to 1998. Treatment from 1998 occurred in the operating room at the time of primary surgical resection employing a dedicated mobile linear accelerator (Mobetron; Intraop Medical, Sunnyvale, CA). Lucite or aluminum cones, 2.1 to $9.5 \mathrm{~cm}$ in interior diameter, were used to deliver electron beams, with energies ranging from 4 to $16 \mathrm{MeV}$. The target volume was encompassed within the $80-90 \%$ isodose line. To ensure coverage of the entire tumor bed and all areas

Table 1 Numbers of evaluated patients who underwent IORT with or without EBRT (IORT \pm EBRT) or EBRT alone in relation to the availability of CT scans. Of the 44 patients with at least one CT scan available, 31 with pre-RT imaging and 25 with both pre- and post-RT imaging were included in the analyses

\begin{tabular}{lllll}
\hline RT cohort & \multicolumn{2}{l}{ Scan availability } & Total \\
\cline { 2 - 4 } & $\begin{array}{l}\text { Pre- and } \\
\text { post-RT }\end{array}$ & $\begin{array}{l}\text { Pre-RT } \\
\text { only }\end{array}$ & $\begin{array}{l}\text { Post-RT } \\
\text { only }\end{array}$ & \\
\hline IORT \pm EBRT & 18 & 2 & 9 & 29 \\
EBRT & 7 & 4 & 4 & 15 \\
Total & 25 & 6 & 13 & 44 \\
\hline
\end{tabular}


deemed at risk of microscopic residual disease, one to four separate IORT fields were used. For the two patients treated in 1993 and 1997, respectively, RT took place in the Radiation Oncology Department. The patients were transported at the time of surgery or reopened on the following day. IORT doses for all patients ranged from 10 to $12.5 \mathrm{~Gy}$, with $90 \%$ of the patients receiving $10 \mathrm{~Gy}$. Shielding of normal tissues not at risk of disease involvement was accomplished by physical exclusion from the radiation field, or by the use of lead sheets when necessary. Patients receiving EBRT were treated during a concurrent time period between 1997 and 2005. EBRT doses ranged from 18 to 24 Gy with $72 \%$ of patients receiving $21.6 \mathrm{~Gy}$. Radiation doses were comparable to those reported elsewhere; however, the threshold dose of blood vessels is unknown [7].

\section{CT technique}

CT examinations were performed with three different helical CT scanners (General Electric Medical Systems, Fairfield, CT). The protocol depended upon the age and weight of the pediatric patient, with the following approximate value ranges: $80-120 \mathrm{kVp}, 60-100 \mathrm{mAs}$, helical slice thickness $3.75-5 \mathrm{~mm}$, pitch $0.562: 1-1.75: 1$, continuous table motion speed $13.75-27.50 \mathrm{~mm} /$ rotation, and reconstruction index $3.75-5 \mathrm{~mm}$. The patients received $2 \mathrm{~cm}^{3} / \mathrm{kg}$ of intravenous Omnipaque 300 (GE Healthcare/Amersham Health, Princeton, NJ).

\section{CT imaging evaluations}

The CT imaging studies selected from the pre- and post-RT scans were those with the closest and latest dates, respectively. For two patients, who were treated with EBRT prior to IORT, the pre-RT CT scan was obtained after the EBRT but before the IORT. Two radiologists in consensus measured the diameter of the abdominal aorta on axial CT scans during the arterial phase of enhancement at four specific locations: primary tumor, celiac artery, superior mesenteric artery and renal arteries. The observed aortic diameters were compared to predicted values for normal children according to the model developed by Fitzgerald et al. [24] whereby the normal aortic diameter is a linear function of age: aortic diameter in centimeters $=0.844+$ $0.0599 \times$ age in years. The observed and predicted changes in aortic diameter at the primary tumor level before and after RT were determined for the following subsets: (1) patients who received IORT with or without EBRT (IORT \pm EBRT) and (2) patients who received EBRT alone (EBRT).

In addition, the diameters of the celiac, superior mesenteric, and renal arteries were evaluated qualitatively on the axial CT scans as normal, focally narrowed, or not visible. A focal narrowing was defined as a decrease in the diameter of more than $50 \%$ with respect to distal portions of the same vessel (in case of visceral branches) or compared to the contralateral side (renal vessels). A quantitative measurement of these branches was not possible because of the small diameters and different orientations of these vessels among studies.

\section{Statistical methods}

The time between CT scans and RT was estimated using the Kaplan-Meier product limit method with distributions between RT groups compared with the log-rank test. To evaluate pre-RT comparability of the two RT patient groups, a chisquared test for categorical variables, $t$-test for independent groups for continuous variables, and the Mann-Whitney test to compare distributions (e.g., age) were performed. The distributions of paired measurements (e.g., predicted and observed aortic diameter) were compared using the paired $t$-test and the nonparametric Wilcoxon matched-pairs test. Pre-RT differences between the observed and predicted aortic diameter at four locations (tumor site, celiac, superior mesenteric, and renal arteries) were compared using analysis of variance (ANOVA) for repeated measures.

The predicted values of aortic growth were determined from a validated equation expressing growth as a linear function of age [1]. For comparison, the predicted and measured values of aortic growth were compared to an agematched control group of 36 children (19 boys, 17 girls) who underwent a CT study for a nononcologic pathology (indications: abdominal pain, appendicitis) and who did not undergo abdominal irradiation. Because predicted values are based upon a linear model, the mean value as a summary measure of a difference or change in growth is more appropriate to present than the median, which is more appropriate for the small samples in this study. Therefore, the data were analyzed using both parametric and nonparametric methods and statistical significance was determined when both tests resulted in a probability value less than 0.05 . For the results presented, the parametric and nonparametric methods always agreed in determining significance and the parametric probability values for tests of means are presented.

\section{Results}

Patient characteristics and RT

The two patient groups (IORT \pm EBRT, and EBRT alone) had comparable disease features at the time of diagnosis and similar follow-up durations from diagnosis (Table 2). The group treated with IORT \pm EBRT included a greater 
Table 2 Patient and disease characteristics at diagnosis

\begin{tabular}{|c|c|c|}
\hline & $\begin{array}{l}\mathrm{IORT} \pm \mathrm{EBRT} \\
(n=20)\end{array}$ & $\begin{array}{l}\text { EBRT } \\
(n=11)\end{array}$ \\
\hline \multicolumn{3}{|c|}{ Follow-up from diagnosis (months) } \\
\hline Median & 23.5 & 19.8 \\
\hline Range & $3.1-122.0$ & $10.9-97.0$ \\
\hline Male, $n(\%)$ & $14(70 \%)$ & $8(73 \%)$ \\
\hline \multicolumn{3}{|l|}{ Age at RT (months) } \\
\hline Mean & 54.7 & 74.6 \\
\hline Median & 32.9 & 54.8 \\
\hline Range & $10.9-247.4$ & $25.1-263.0$ \\
\hline \multicolumn{3}{|l|}{ Age groups $(n)$} \\
\hline$<24$ months & 5 & 0 \\
\hline$>72$ months & 4 & 4 \\
\hline \multicolumn{3}{|l|}{ Stage, $n(\%)^{\mathrm{a}}$} \\
\hline II & $1(5 \%)$ & \\
\hline III & $5(25 \%)$ & $4(36 \%)$ \\
\hline IV & $13(65 \%)$ & $7(64 \%)$ \\
\hline IVs & $1(5 \%)$ & - \\
\hline \multicolumn{3}{|l|}{ N-MYC amplification, $n(\%)$} \\
\hline Yes & $3(15 \%)$ & $4(36 \%)$ \\
\hline No & $8(40 \%)$ & - \\
\hline Unknown & $9(45 \%)$ & $7(64 \%)$ \\
\hline \multicolumn{3}{|l|}{ Surgery, $n(\%)$} \\
\hline Gross total resection & $14(70 \%)$ & $9(82 \%)$ \\
\hline Subtotal resection & $6(30 \%)$ & - \\
\hline Unknown & - & $2(18 \%)$ \\
\hline \multicolumn{3}{|c|}{ Pathologic lymph node involvement, $n(\%)$} \\
\hline Yes & $7(35 \%)$ & $4(36 \%)$ \\
\hline No & $2(10 \%)$ & $1(9 \%)$ \\
\hline Unknown & $11(55 \%)$ & $6(55 \%)$ \\
\hline $\begin{array}{l}\text { Hematopoietic Stem Cell } \\
\text { Transplant, } n(\%)\end{array}$ & $13(65 \%)$ & $10(91 \%)$ \\
\hline
\end{tabular}

${ }^{a}$ International Neuroblastoma Staging System.

number of patients who were younger than 24 months at diagnosis $(P=0.06)$, at the time of the pre-RT scan $(P=0.07)$, and at the time of RT $(P=0.07)$, compared with the group of patients treated with EBRT alone. Although these borderline statistically significant differences were observed when the patients were categorized according to age ( $<24$ vs. $>24$ months), there was no difference in the overall distributions of age between the RT groups.

For each RT group, the measured mean aortic diameter was significantly lower than the predicted normal value and the normal aorta of nonirradiated control patients ( $P<0.0001$ for each comparison). For both RT groups, all pair-wise comparisons among the four aortic measurements as well as the predicted normal values were highly correlated $(P<0.001)$ for each comparison for the entire study sample and within each RT subset. There was no significant difference in the mean prescan measurements between the two RT groups overall at each of the four locations (overall ANOVA for repeated measures $P=0.71$; $P>0.44$ for each location). These pre-RT measurements were obtained prior to any RT, so correlations at the four measurement locations for the entire study sample of irradiated patients could be evaluated together. Therefore, the remainder of this analysis focuses on aortic measurements at the primary tumor site.

Pre-RT aortic diameter

The median time from the pre-RT CT scan to IORT \pm EBRT was 1.1 months with 19 of 20 patients treated within 4.5 months of the baseline scan. The median duration from the pre-RT scan to EBRT was 0.5 months with 8 of 11 patients treated within 1 month of the baseline scan. The mean observed aortic diameter was $0.74 \mathrm{~cm}$ (range 0.40 $1.30 \mathrm{~cm}$ ) before treatment with IORT \pm EBRT and $0.93 \mathrm{~cm}$ (range $0.51-1.76 \mathrm{~cm}$ ) before treatment with EBRT alone. The aortic diameter of both groups was significantly smaller than the predicted normal aortic diameter (mean difference: IORT \pm EBRT $-0.37, P<0.0001$; EBRT -0.27 , $P<0.0001$; Fig. 1).

\section{Post-RT aortic diameter and aortic growth}

For the 25 patients with both pre- and post-RT CT scans, the median CT follow-up time between the pre-RT and post-RT CT scans did not differ significantly between the IORT \pm EBRT group (median 22.1 months, range 0.4-72.5 months) and the EBRT group (median 27.8 months, range 9.2-70.6 months). There was also no difference in age at the time of the postRT scan (median 5.7 years, range 1.3-22.6 years in the IORT \pm EBRT group; median 6.6 years, range 3.2-25.9 years in the EBRT group) so that a similar distribution of the observed aortic diameters would be expected for the two RT groups.

The observed post-RT aortic diameter at the primary tumor site was significantly smaller than the predicted value for both the IORT \pm EBRT group $(P<0.0001)$ and the EBRT group (mean $P=0.003$ ). The post-RT mean predicted aortic diameter was $1.25 \mathrm{~cm}$ (range $0.92-2.20 \mathrm{~cm}$ ) for the IORT \pm EBRT group and $1.38 \mathrm{~cm}$ (range 1.03-2.40 cm) for the EBRT group. The post-RT mean observed aortic diameter was $0.80 \mathrm{~cm}$ (range $0.50-1.48 \mathrm{~cm}$ ) for the IORT \pm EBRT group and $0.82 \mathrm{~cm}$ (range $0.54-1.26 \mathrm{~cm}$ ) for the EBRT group. There was no significant difference between the two RT groups $(P<0.05$; Fig. 1$)$.

Patients who received RT showed no significant change in aortic diameter between the pre-RT and post-RT CT scans (mean change: IORT \pm EBRT $0.03 \mathrm{~cm}, P=0.39$; EBRT $-0.10 \mathrm{~cm}, P=0.22$; Fig. 2). 
Pre-RT: Difference From Predicted by RT

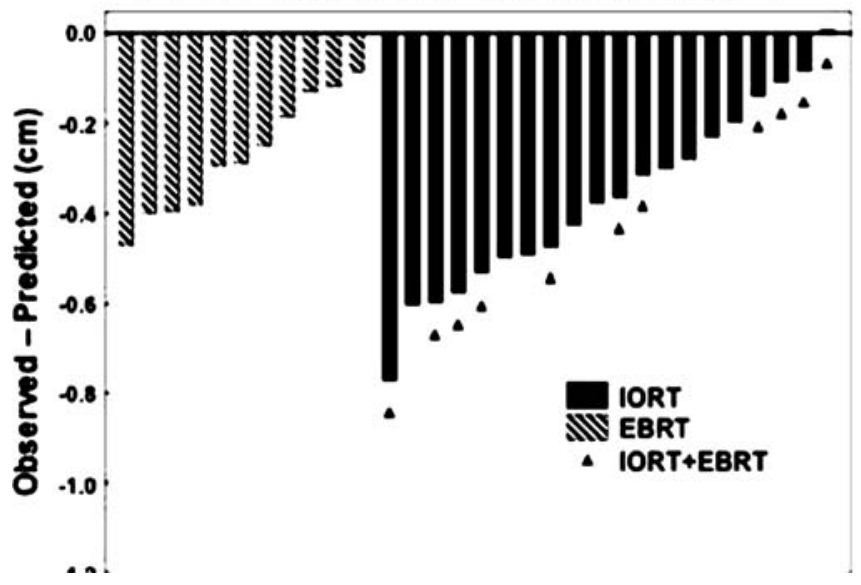

Fig. 1 Difference between observed and predicted aortic diameter for patients treated with EBRT (hatched bars) or IORT with or without EBRT (IORT \pm EBRT, solid bars). Before RT, only one patient in the entire study sample had an aortic diameter at least as large as the predicted value. All other RT patients showed significantly smaller aortic diameters than predicted $(P<0.0001)$.

Visceral and renal branches of the aorta

Abnormalities of the vascular branches of the aorta were documented after RT in five patients treated with IORT+EBRT and in two patients treated with EBRT. Patients treated with IORT \pm EBRT demonstrated narrowing of the celiac trunk (one patient), superior mesenteric artery (two), and renal arteries (two) as well as asymmetric perfusion of the kidneys (one). Three of these patients had clinical symptoms of MAS. Two patients treated with EBRT showed a narrowing of the celiac trunk (one) and asymmetric perfusion of the kidneys (two). However, at the

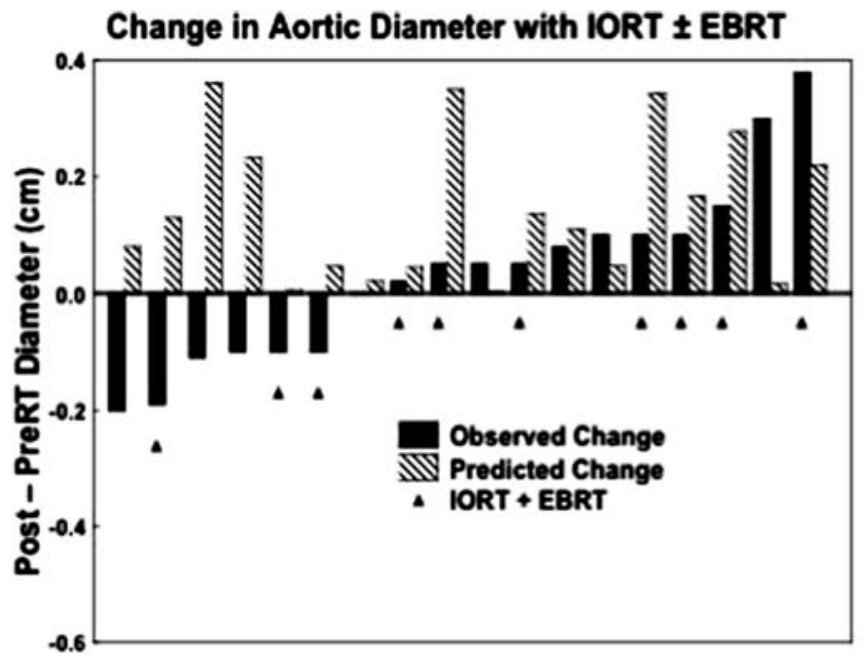

Fig. 2 Aortic diameters before and after RT. There are two bars for each patient indicating the observed (solid bars) and predicted normal (hatched bars) changes in aortic diameter. The negative bars indicate a
Post-RT: Difference From Predicted by RT

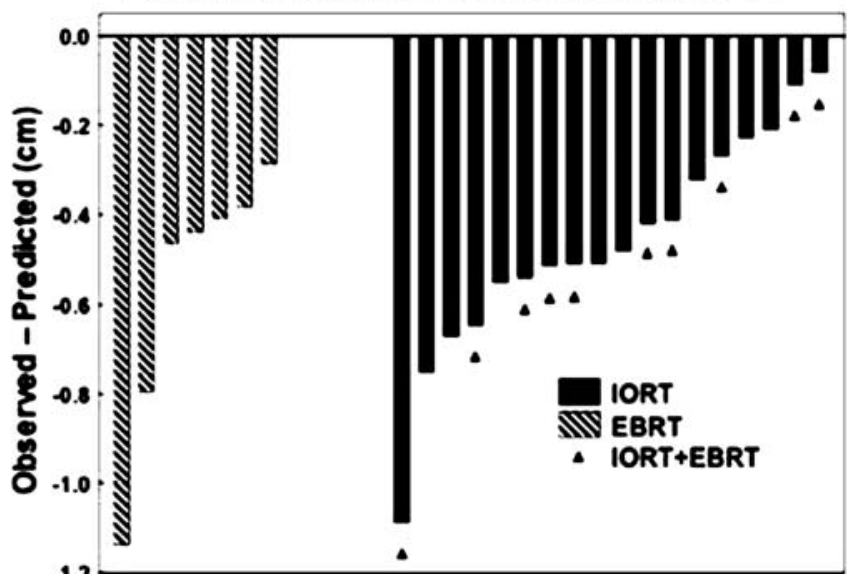

After RT, the aortic diameters were smaller than predicted for all patients (IORT \pm EBRT $P<0.0001$, EBRT $P=0.003$ ). Only two patients treated with IORT+EBRT had measurements within $0.2 \mathrm{~cm}$ of their predicted value. The discrepancy between observed and predicted aortic diameters increased after RT

time of this report neither of these two patients had clinical signs or symptoms referable to these radiographic findings.

\section{Clinical follow-up after RT}

At the time of this report, 8 of the 20 patients treated with IORT \pm EBRT had shown recurrence and had died. Among the 11 patients treated with EBRT, 7 had shown recurrence, of whom 5 have died. Seven patients treated with IORT \pm EBRT had one or more clinical long-term complications that included hypertension (five), MAS (three), and death due to mesenteric ischemia (one; Figs. 3 and 4). The one patient who died of mesenteric ischemia did not

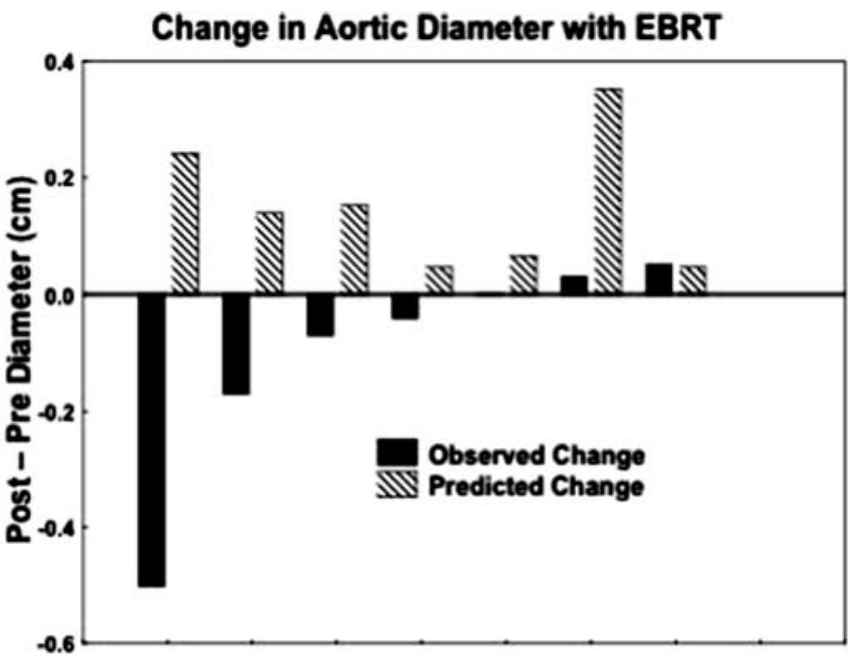

decrease in aortic diameter. Both RT groups showed significantly less growth of the aorta than expected $(P<0.05)$ 

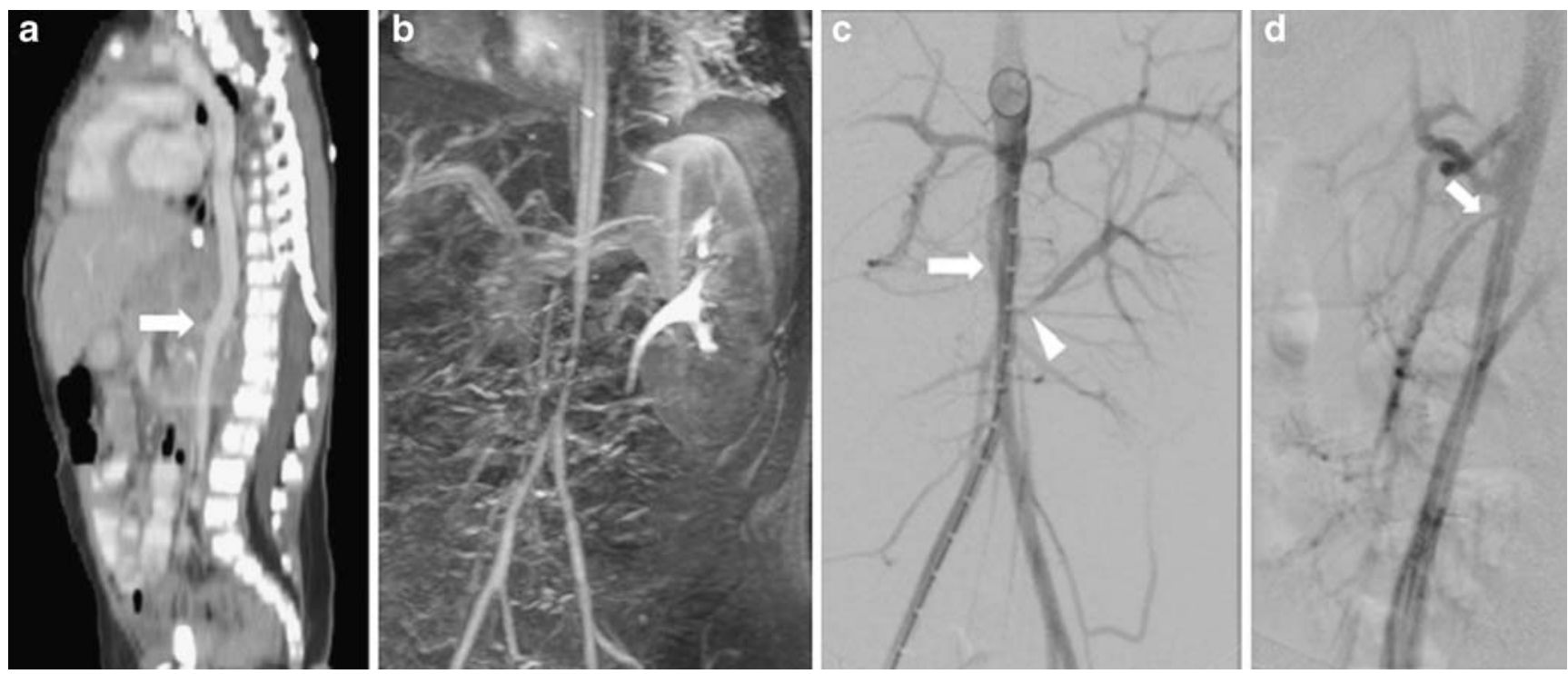

Fig. 3 Imaging studies of a girl with stage IV neuroblastoma who developed hypertension and abdominal pain 5 years after right nephrectomy, chemotherapy and IORT. a Sagittal contrast-enhanced CT image prior to treatment shows a normal caliber of the abdominal aorta (white arrow), which is encased by tumor. b Gadoliniumenhanced abdominal MR angiogram 5 years later demonstrates a markedly narrowed infrarenal abdominal aorta to $3 \mathrm{~mm}$ in diameter. c Conventional angiography confirms narrowing of the abdominal aorta (arrow) and proximal left renal artery (arrowhead). d Lateral view from conventional angiography shows additional narrowing of the proximal superior mesenteric artery (arrow)
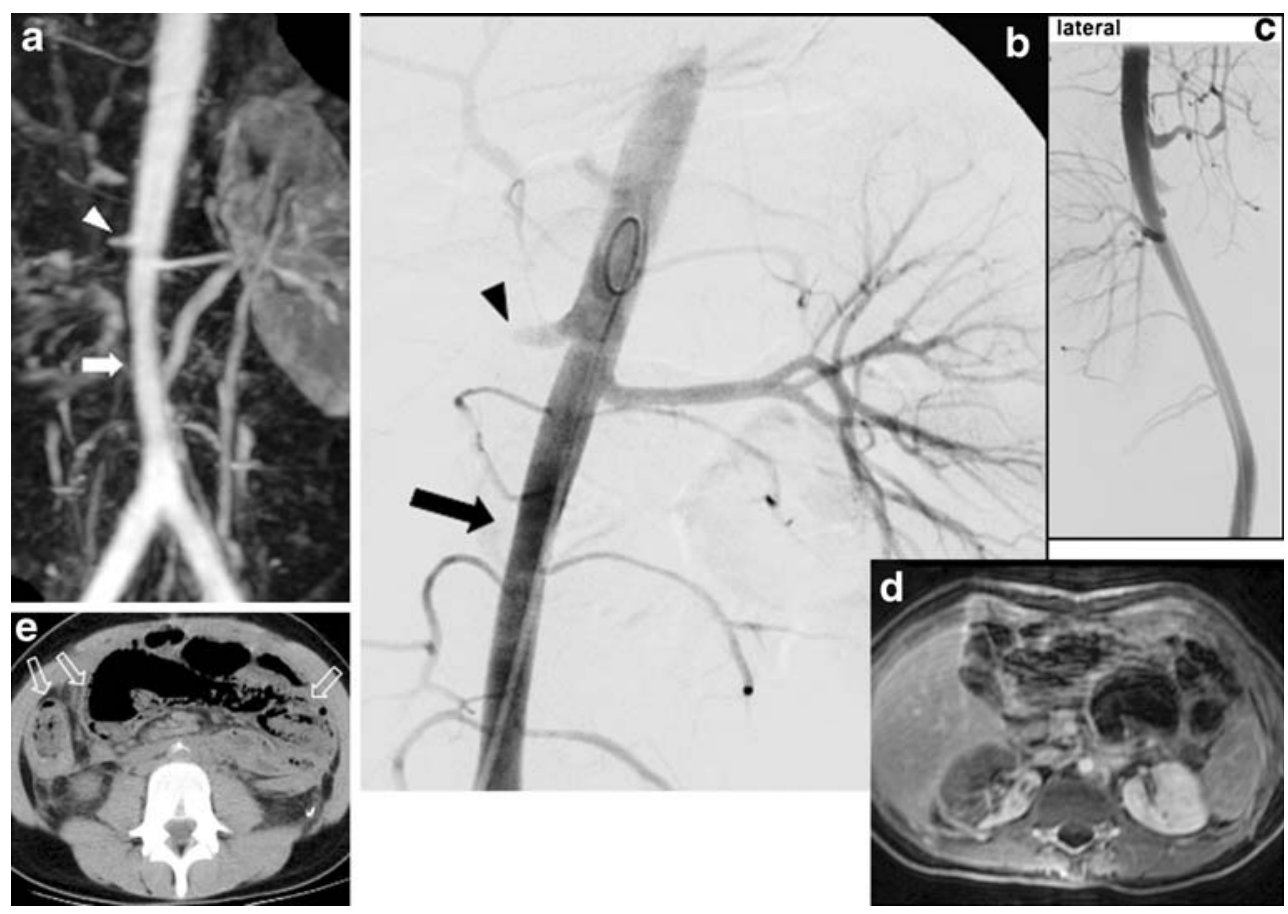

Fig. 4 Imaging studies of a boy with stage IV neuroblastoma who developed hypertension and severe bowel ischemia 12 years after treatment with surgery, chemotherapy, IORT, total body irradiation and bone marrow transplantation. The patient underwent aortic bypass surgery, but died shortly afterwards from complications of mesenteric ischemia. a-c MR angiography and conventional angiography show complete occlusion of the superior and inferior mesenteric arteries as well as a complete occlusion of the right renal artery (arrowhead) near their origins from the abdominal aorta. There is also a focal stenosis of the celiac trunk near its origin with complete occlusion of the splenic artery. The caliber of the infrarenal aorta is mildly decreased (arrow). d Axial T1-W MR image after intravenous Gd-DTPA injection shows a large right infarct of the right kidney as a result of occlusion of the right renal artery. e CT image demonstrates extensive pneumatosis of small bowel loops due to mesenteric ischemia 

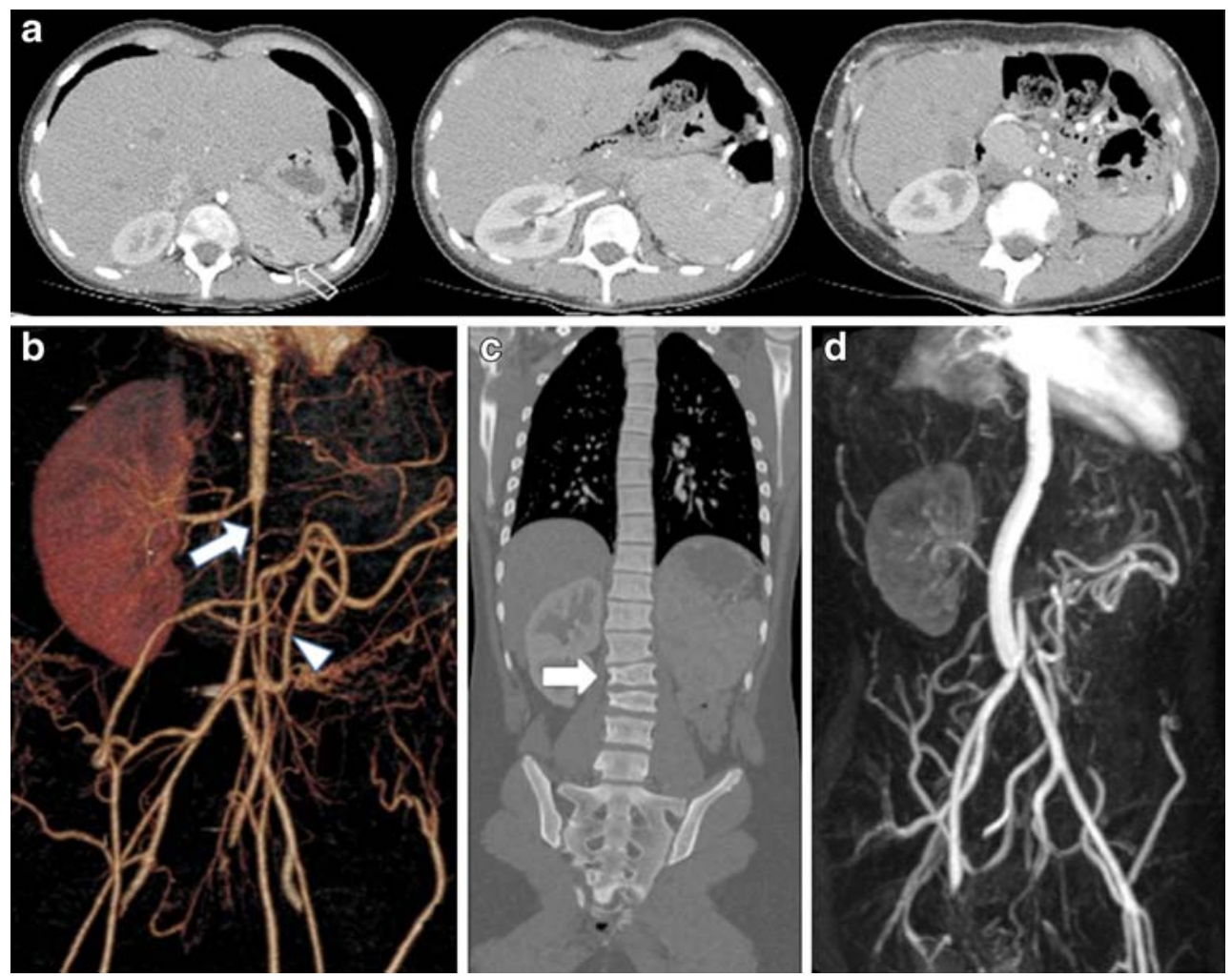

Fig. 5 Imaging studies of a girl with stage IV neuroblastoma who developed hypertension and abdominal pain 7 years after tumor surgery, chemotherapy and IORT. a, b Axial contrast-enhanced CT angiograms (a) and 3-D reconstruction (b) show an extremely narrowed infrarenal aorta (arrow), as well as absent celiac, superior mesenteric and left renal arteries. The left kidney is not visible due to marked atrophy (a open arrow). Multiple abdominal collateral vessels are visualized (arrowhead). The right renal artery demonstrates mild proximal stenosis. $\mathbf{c}$ Coronal reformat shows additional deformities of the lumbar vertebrae (arrow). d MR angiogram after treatment with right renal artery angioplasty and right aortorenal bypass surgery shows a widely patent aortic bypass graft, conduit and anastomoses, and persistent absent flow in the celiac trunk; hepatic artery flow is maintained via collaterals show symptoms of MAS. The average time between RT and the diagnosis of MAS was 9 years (range 5 to 14 years). All three patients with MAS underwent aortorenal bypass surgery and one patient also required a thoracic to infrarenal aortic bypass. All three patients with MAS demonstrated growth deformities of the upper lumbar vertebrae (Fig. 5). Of the seven patients with complications, four were less than 24 months old and two were older than 72 months at the time of IORT \pm EBRT. Therefore, there is no clear evidence of a difference in complications due to age at the time of IORT \pm EBRT. No patient in the EBRT group reported clinical symptoms of MAS or other signs of visceral ischemia. There was no apparent association between the degree of aortic growth inhibition and the occurrence of clinical complications.

\section{Discussion}

Both groups of patients treated with RT (IORT \pm EBRT and EBRT) had significantly smaller measured aortic diameters than the predicted age-adjusted normal aortic diameters before RT. Patients with neuroblastoma who received abdominal RT had a significant decrease in aortic growth. Several patients treated with IORT \pm EBRT developed serious complications, including MAS. MAS is a rare entity most prevalent among adolescents and young adults [22, 25]. Sen et al. [23] were the first to describe it in 1963 as a severe narrowing of the proximal abdominal aorta. Clinically the main presenting symptom is hypertension and symptoms may variably include severe headache, nosebleeds, chest pain, cardiac failure and kidney failure [26]. The etiology remains unknown $[22,25,26]$. The histology of MAS is described as nonspecific dysplastic, fibrotic changes lacking signs of inflammation, or necrosis [27, 28].

The biological effect of radiation on the vasculature is well documented for the coronary arteries and microvasculature, but otherwise is not well documented [29, 30]. Gillette et al. [20] described the response of canine aorta and branch arteries to experimental IORT and reported a narrowing of the aorta on aortography and a thickening of the intima on histopathology, occurring more than 5 years 
after IORT [30]. From these studies, it is evident that follow-up time is an important factor when fully assessing toxicities of IORT and EBRT.

As a result of continuing therapeutic advances, children with cancer are surviving longer than in previous decades, rendering long-term follow-up studies essential for optimal treatment and continued care. Pediatric studies reviewing the effects of IORT in children involve follow-up ranges of 6 to 101 months after RT. These reports suggest that IORT improves local control of disease with high doses of radiation and that complications at doses used were trivial.

It should be noted that the populations studied were small and follow-up periods relatively short $[7,11,12,14$, $17,18]$. Of note, there was no radiological documentation in the aforementioned studies of MAS. Our findings are in accordance with reports of a patient with renal artery stenosis, a patient with mesenteric artery ischemia, and a patient with hypertension after IORT for neuroblastoma [7, $11,12]$. In the adult population, analyses of side effects in patients surviving more than 5 years after IORT have identified significant vascular occlusion resulting in irreversible functional damage requiring aggressive management $[15,31]$. CT imaging studies were used because all patients underwent $\mathrm{CT}$ at diagnosis and subsequently to monitor disease progression and/or therapeutic response, thus allowing this retrospective review. A gold-standard modality to measure the abdominal aorta does not exist. CT and US are commonly used; however, they are both subject to significant interobserver variability. Research evaluating the size of the pediatric aorta is extremely limited and there are no recognized age-adjusted reference values for either CT or US. Fitzgerald et al. [24] have conducted the only study that has evaluated the pediatric aortic diameter on CT. Several US studies have shown independent pediatric abdominal aortic diameter nomograms in relation to various factors such as age, gender, weight, height, body mass index and body surface area without consensus on variations in relation to sex.

Several limitations of this retrospective study have to be recognized. First, the exact etiology of the decreased aortic size and growth was uncertain, although RT is certainly plausible; yet other confounding variables must be considered. The aggressive nature of the tumor itself may have contributed to the observed decrease in pre-RT aortic diameter, and residual tumor after gross surgical resection may have caused the subsequent decreased rate of aortic growth. However, MAS was documented in four patients in remission. In addition, a specific biochemical profile/growth factor, chemotherapy, surgery, hematopoietic stem cell transplantation and/or unknown pathologic contributing factor could have contributed to the impaired aortic size and growth, and development of MAS. Evaluation of aortic size and growth in a matched cohort of neuroblastoma patients not treated with RT would provide additional information. Second, we could not determine a direct correlation between the degree of decrease in aortic caliber and vascular complications. This may have been due to potential additional contributing factors such as impairments at the level of major abdominal arteries or microvessels and the small size of the study cohort. Conversely, some of our results may describe complications not related to radiation or may be confounded by additional therapeutic procedures such as surgery. All patients who received IORT did so for gross residual disease or for tumors deemed unresectable, and so many had worse tumors, explaining the higher rate of complications, i.e. these patients may have developed the problems from the tumor and/or surgery itself. Finally, the $\mathrm{CT}$ imaging protocol used was not defined and institutional access to CT scans limited the cohort size and median follow-up for aortic growth. A prospective large study of comparable cohorts of patients as to the extent of disease treated with designated radiation modalities and careful uniform imaging at defined time points would be necessary to answer some of these questions.

Medical management for MAS in the pediatric population is preferred until the child has ceased growing so as to prevent a second surgery to accommodate the growth. The treatment of choice for MAS is now either a one-stage reconstructive prosthetic or autologous venous surgical arterial bypass graft.

In conclusion, limited aortic growth after RT in patients with neuroblastoma diagnosed on CT scans may be the first sign of MAS. Radiologists and clinicians should be aware of the possibility of such a diagnosis and the important consequences that arise in regard to patient management. Long-term CT follow-up studies including coronal reconstruction images are required in pediatric patients who receive IORT and EBRT to assess potential toxicities and to determine the exact complication rate.

Open Access This article is distributed under the terms of the Creative Commons Attribution Noncommercial License which permits any noncommercial use, distribution, and reproduction in any medium, provided the original author(s) and source are credited.

\section{References}

1. Siegel MJ, Ishwaran H, Fletcher BD et al (2002) Staging of neuroblastoma at imaging: report of the radiology diagnostic oncology group. Radiology 223:168-175

2. Kushner BH, LaQuaglia MP, Cheung NK (1993) Rethinking management of localized neuroblastoma. J Clin Oncol 11:18321834

3. Kushner BH (2004) Neuroblastoma: a disease requiring a multitude of imaging studies. J Nucl Med 45:1172-1188

4. Matthay KK (1997) Neuroblastoma: biology and therapy. Oncology (Williston Park) 11:1857-1866 discussion 1869-1872, 1875 
5. Matthay KK, Villablanca JG, Seeger RC et al (1999) Treatment of high-risk neuroblastoma with intensive chemotherapy, radiotherapy, autologous bone marrow transplantation, and 13-cis-retinoic acid. Children's Cancer Group. N Engl J Med 341:1165-1173

6. Castleberry RP, Kun LE, Shuster JJ et al (1991) Radiotherapy improves the outlook for patients older than 1 year with Pediatric Oncology Group stage C neuroblastoma. J Clin Oncol 9:789-795

7. Haas-Kogan DA, Fisch BM, Wara WM et al (2000) Intraoperative radiation therapy for high-risk pediatric neuroblastoma. Int $\mathrm{J}$ Radiat Oncol Biol Phys 47:985-992

8. Hawkins MM (1990) Second primary tumors following radiotherapy for childhood cancer. Int J Radiat Oncol Biol Phys 19:1297-1301

9. Meadows AT (1989) Second malignant neoplasms in childhood cancer survivors. J Assoc Pediatr Oncol Nurses 6:7-11

10. Suleiman $\mathrm{OH}$ (2004) Radiation doses in pediatric radiology: influence of regulations and standards. Pediatr Radiol 34(Suppl 3): S242-S246

11. Leavey PJ, Odom LF, Poole M et al (1997) Intraoperative radiation therapy in pediatric neuroblastoma. Med Pediatr Oncol $28: 424-428$

12. Zachariou Z, Sieverts H, Eble MJ et al (2002) IORT (intraoperative radiotherapy) in neuroblastoma: experience and first results. Eur J Pediatr Surg 12:251-254

13. Sindelar WF, Tepper JE, Kinsella TJ et al (1994) Late effects of intraoperative radiation therapy on retroperitoneal tissues, intestine, and bile duct in a large animal model. Int J Radiat Oncol Biol Phys 29:781-788

14. Aitken DR, Hopkins GA, Archambeau JO et al (1995) Intraoperative radiotherapy in the treatment of neuroblastoma: report of a pilot study. Ann Surg Oncol 2:343-350

15. Azinovic I, Calvo FA, Puebla F et al (2001) Long-term normal tissue effects of intraoperative electron radiation therapy (IOERT): late sequelae, tumor recurrence, and second malignancies. Int $\mathrm{J}$ Radiat Oncol Biol Phys 49:597-604

16. Sindelar WF, Kinsella TJ (2003) Normal tissue tolerance to intraoperative radiotherapy. Surg Oncol Clin N Am 12:925-942

17. Kuroda T, Saeki M, Honna T et al (2003) Clinical significance of intensive surgery with intraoperative radiation for advanced neuroblastoma: does it really make sense? J Pediatr Surg 38:1735-1738
18. Haase GM, Meagher DP Jr, McNeely LK et al (1994) Electron beam intraoperative radiation therapy for pediatric neoplasms. Cancer 74:740-747

19. Merchant TE, Zelefsky MJ, Sheldon JM et al (1998) High-dose rate intraoperative radiation therapy for pediatric solid tumors. Med Pediatr Oncol 30:34-39

20. Gillette EL, Powers BE, McChesney SL et al (1989) Response of aorta and branch arteries to experimental intraoperative irradiation. Int J Radiat Oncol Biol Phys 17:1247-1255

21. Lewis VD 3rd, Meranze SG, McLean GK et al (1988) The midaortic syndrome: diagnosis and treatment. Radiology 167:111-113

22. Panayiotopoulos YP, Tyrrell MR, Koffman G et al (1996) Midaortic syndrome presenting in childhood. Br J Surg 83:235240

23. Sen PK, Kinare SG, Engineer SD et al (1963) The middle aortic syndrome. Br Heart J 25:610-618

24. Fitzgerald SW, Donaldson JS, Poznanski AK (1987) Pediatric thoracic aorta: normal measurements determined with CT. Radiology 165:667-669

25. Lee LC, Broadbent V, Kelsall W (2000) Neuroblastoma in an infant revealing middle aortic syndrome. Med Pediatr Oncol $35: 150-152$

26. Sumboonnanonda A, Robinson BL, Gedroyc WM et al (1992) Middle aortic syndrome: clinical and radiological findings. Arch Dis Child 67:501-505

27. Riemenschneider TA, Emmanouilides GC, Hirose F et al (1969) Coarctation of the abdominal aorta in children: report of three cases and review of the literature. Pediatrics 44:716-726

28. Poulias GE, Skoutas B, Doundoulakis N et al (1990) The midaortic dysplastic syndrome. Surgical considerations with a 2 to 18 year follow-up and selective histopathological study. Eur $\mathrm{J}$ Vasc Surg 4:75-82

29. Veinot JP, Edwards WD (1996) Pathology of radiation-induced heart disease: a surgical and autopsy study of 27 cases. Hum Pathol 27:766-773

30. Dubois JB (1997) Late effects of intraoperative radiotherapy. Cancer Radiother 1:817-822

31. Shimizu Y, Yasui K, Fuwa N et al (2005) Late complication in patients undergoing pancreatic resection with intraoperative radiation therapy: gastrointestinal bleeding with occlusion of the portal system. J Gastroenterol Hepatol 20:1235-1240 